\section{Parental Perceptions about Required Influenza Immunization of Pediatric Healthcare Personnel}

\author{
W. Matthew Linam, MD, MS; ${ }^{1,2}$ \\ Craig H. Gilliam, BSMT, CIC; ${ }^{2}$ \\ Michele Honeycutt, RN, BSN, CIC; ${ }^{2}$ \\ Christy Wisdom, RN, BSN, CIC; ${ }^{2}$ \\ Christopher J. Swearingen, $\mathrm{PhD} ;^{3}$ José R. Romero, $\mathrm{MD}^{1}$
}

Annual influenza vaccination is recommended for all healthcare personnel (HCP). During 2010-2011, a cross-sectional design was used to survey 372 parents of hospitalized children regarding their influenza vaccination perceptions. Independent of their feelings regarding vaccine safety and efficacy, $76 \%$ of parents felt that annual influenza vaccination should be required for HCP.

Infect Control Hosp Epidemiol 2014;35(10):1301-1303

Influenza transmission between healthcare personnel (HCP) and patients is well documented. ${ }^{1}$ Influenza vaccination is the most effective way to prevent influenza infection in healthcare settings. The Advisory Committee on Immunization Practices (ACIP) recommends that all HCP receive influenza vaccination annually. ${ }^{1}$ Despite this recommendation, annual HCP vaccination rates before 2008 were below $45 \% .{ }^{1,2}$ Strategies to improve HCP vaccination rates have included educational campaigns, increasing vaccine availability, prioritizing influenza vaccination within organizations, and use of mandatory declination forms. ${ }^{2}$ As a result, vaccination coverage of HCP has gradually increased to $72 \%$ during $2012-2013 .{ }^{3}$

To consistently reach high rates of HCP influenza vaccination, many hospitals now require annual influenza vaccination of their staff. Hospitals implementing mandatory HCP vaccination have achieved over $95 \%$ vaccination coverage, ${ }^{3,4}$ whereas hospitals that have not implemented such policies have achieved only $78 \%$ coverage. $^{3}$

At Arkansas Children's Hospital (ACH), HCP influenza vaccination coverage between 2007 and 2010 ranged from $65 \%$ to $85 \%$. In $2011, \mathrm{ACH}$ proposed an annual HCP influenza vaccination requirement. There are no data regarding parental perception concerning the importance of HCP influenza vaccination or whether parental perceptions regarding the safety and efficacy of influenza vaccines influence their perception about HCP vaccination. This study investigates parent perceptions regarding the importance, safety, and efficacy of influenza vaccination as they relate to the need for annual HCP influenza vaccination.

\section{METHODS}

This institutional review board-approved study was performed at ACH, a 370-bed freestanding children's hospital.
A cross-sectional survey of parents and guardians of children hospitalized at ACH was performed over three 4-week periods from Fall 2010 through Summer 2011. An internally developed 16-question, self-administered paper questionnaire assessed the perceived importance of influenza vaccination for parents, children, and HCP; the perceived safety and efficacy of influenza vaccines (defined as yes, no, or unsure); and parent demographic information. Additional data collected included study population disposition and patient demographic data and clinical features.

Surveys were administered during business hours each weekday during the following 3 time periods: Fall 2010 (before the influenza season), Winter 2011 (during the influenza season), and Summer 2011 (after the influenza season). Each weekday morning, all new inpatients from the preceding 24 hours (or the preceding 72 hours on Mondays) were identified from the admissions database. If the patient had been discharged from the hospital, no attempts were made to contact them. Survey administrators made reasonable attempts to enroll 1 parent or guardian for each patient in the 24 hours after admission. Parents and guardians between 18 and 65 years of age were included. If the patient was critically ill and enrollment seemed inappropriate, the survey administrator could exclude the parent or guardian from the study. If a parent or guardian was unavailable, no attempts to contact a parent or guardian were made on subsequent days of hospitalization.

Demographic and survey responses were summarized by study time period; categorical data were summarized by frequency and percentage, whereas continuous data were summarized as median and range (minimum and maximum). Similarity between the cohort's demographic and admittance data and the hospital data for the same time period was estimated using equivalence tests that assumed a $20 \%$ margin. ${ }^{5}$ Differences between time periods were investigated using $\chi^{2}$ test (Fisher exact test if assumptions failed) for categorical data and Kruskal Wallis test for continuous and ordinal data. Firth-conditional as well as logistic regression models estimated any association between outcomes (HCP vaccination importance and requirement) and parent perception of vaccine importance for themselves or their children ("yes" vs "no" and "unsure" responses) while adjusting for vaccine safety, vaccine efficacy, insurance status, race, intensive care unit (ICU) admission, and parent's education level.

\section{RES U L T S}

A total of 372 parents were enrolled and completed questionnaires (Fall 2010: 132; Winter 2011: 125; Summer 2011: 115). This represented $11 \%$ of all admitted patients during the study time periods. Of the parents and guardians not enrolled, $50.5 \%$ were not approached, $14 \%$ had been dis- 
charged, $23 \%$ were excluded and only $0.7 \%$ declined enrollment. The study sample was equivalent to the hospitalized population in terms of white parents and guardians as well as Medicaid and privately insured patients. The sample population was slightly older (sample median age was 5.5 years compared with 3.0 years for the admitted population), included more female patients, and included fewer black parents and guardians and fewer ICU admissions compared with the hospitalized population.

No statistical differences between time periods were estimated for patient age, sex, or ICU hospitalization status or parent age, sex, race, or education (Table 1). Insurance type and parent's ethnicity differed between time periods.

There were no significant differences between study periods for survey responses; therefore, responses from the 3 time periods were combined. Vaccinating their children against influenza was important for $75 \%$ of parents, but self-vaccination was important for only $68 \%$ of parents. The majority (71\%) believed influenza vaccines were safe, but only $57 \%$ thought that they were effective. Eighty-eight percent of parents felt that HCP should receive an annual influenza vaccination; $76 \%$ thought that it should be required.

Parent's belief that self-vaccination was important was associated with increased odds of both reported importance for HCP influenza vaccination (Firth odds ratio [OR], $31.1[95 \%$ confidence interval (CI), 5.1-188.8]; $P<.001)$ and HCP vac- cination requirement (OR, 4.8 [95\% CI, 2.1-11.1]; $P<.001$ ). Moreover, parent's belief that their child's vaccination was important was also associated with increased odds of reported importance of HCP vaccination requirement (OR, 3.2 [95\% CI, 1.4-7.2]; $P=.005)$. These associations were independent of the nonsignificant covariates race, insurance, education, ICU admission, safety, and effectiveness beliefs.

\section{DISCUSSION}

This is, to our knowledge, the first study to evaluate parental perceptions about HCP influenza vaccination. We found that the vast majority $(88 \%)$ of parents and guardians of hospitalized children thought that HCP should receive annual influenza vaccinations, and $76 \%$ thought that such vaccinations should be required.

On the basis of the Centers for Disease Control and Prevention estimates, only $72 \%$ of HCP reported receiving influenza vaccination during the 2012-2013 influenza season. ${ }^{3}$ In 2010, the Society of Healthcare Epidemiology of America endorsed a policy requiring HCP annual influenza vaccination. ${ }^{4}$ Since that time, many hospitals now require annual influenza vaccination as a condition of HCP employment, resulting in influenza vaccination coverage greater than $95 \% .{ }^{3,4}$ With only $62 \%$ of HCP reporting that their hospital employers require influenza vaccination, a significant number

T A B LE 1. Summary Demographic Data of 372 Parents and Their Hospitalized Children Surveyed During 2010-2011

\begin{tabular}{|c|c|c|c|c|c|}
\hline Variable & Fall 2010 & Winter 2011 & Spring 2011 & Total & $P$ \\
\hline No. of patients & 132 & 125 & 115 & 372 & \\
\hline Age, median (range), years & $5.6(0-18.9)$ & $4.6(0-18.6)$ & $6.7(0-19.5)$ & $5.5(0-19.5)$ & .781 \\
\hline Female sex & $110(83)$ & $104(83)$ & $85(74)$ & $299(80)$ & $.110^{\mathrm{a}}$ \\
\hline ICU hospitalization & $15(11)$ & $17(14)$ & $15(13)$ & $47(13)$ & $.854^{\mathrm{a}}$ \\
\hline Insurance & & & & & $.006^{\mathrm{b}}$ \\
\hline Medicaid & $95(72)$ & $77(62)$ & $67(58)$ & $239(64)$ & \\
\hline Private & $31(23)$ & $46(37)$ & $36(31)$ & $113(31)$ & \\
\hline Self-pay & $6(5)$ & $2(1)$ & $12(11)$ & $20(5)$ & \\
\hline Parent age, median (range), years & $32(18-59)$ & $33(17-58)$ & $34(18-57)$ & $33(17-59)$ & .507 \\
\hline Parent female sex & $53(40)$ & $51(41)$ & $52(45)$ & $156(42)$ & $.688^{\mathrm{a}}$ \\
\hline Parent race & & & & & $.909^{\mathrm{b}}$ \\
\hline White & $102(77)$ & $96(78)$ & $87(77)$ & $285(78)$ & \\
\hline Black & $24(18)$ & $19(16)$ & $21(19)$ & $64(17)$ & \\
\hline Other & $6(5)$ & $8(7)$ & $5(4)$ & $19(5)$ & \\
\hline Parent Hispanic & $6(5)$ & $16(14)$ & $8(8)$ & $30(9)$ & $.050^{\mathrm{b}}$ \\
\hline Parent education & & & & & $.117^{\mathrm{c}}$ \\
\hline Some high school & $14(11)$ & $10(8)$ & $8(7)$ & $32(9)$ & \\
\hline High school graduate & $50(38)$ & $39(32)$ & $34(30)$ & $123(34)$ & \\
\hline Some college/vocation & $44(33)$ & $46(38)$ & $39(35)$ & $129(35)$ & \\
\hline College graduate & $18(14)$ & $18(15)$ & $24(21)$ & $60(16)$ & \\
\hline Postgraduate & $6(5)$ & $9(7)$ & $7(6)$ & $22(6)$ & \\
\hline
\end{tabular}

Nоте. Unless otherwise indicated, data are no. (\%) of patients or parents and guardians, and $P$ values are determined by Wilcoxon-Mann-Whitney test.

${ }^{\mathrm{a}} \chi^{2}$ test reported.

b Fisher exact test reported.

c Wilcoxon-Mann-Whitney reported for ordinal data. 
of hospitals have yet to adopt this recommendation. ${ }^{3} \mathrm{HCP}$ resistance to vaccination is an important barrier to hospitals requiring annual influenza vaccination and must be overcome to facilitate successful implementation. ${ }^{4}$

Perceived social norm has been cited as an independent predictor of HCP vaccinating themselves and parents vaccinating their children against influenza. ${ }^{6,7}$ Social norms are based on the shared beliefs held by the majority of individuals and provide informal understandings that guide society's behavior. Descriptive norms refer to the behavior of most people in a certain situation. ${ }^{8}$ Injunctive norms refer to whether a behavior is approved by a particular group and are powerful motivators of behavior. ${ }^{8}$ In our study, the majority of parents expected HCP to be vaccinated against influenza, suggesting this is an injunctive norm. The perceived prevalence and approval of protective health behaviors are often underestimated, leading to engagement in unhealthy behaviors. ${ }^{9}$ Correcting misperceptions of perceived norms has resulted in increased healthy behavior. ${ }^{8}$ Increasing HCP awareness that annual influenza vaccination is expected by parents may promote HCP vaccination and facilitate hospitals adopting a policy of required influenza vaccination of HCP.

This study has several limitations. Parent and guardian responses were obtained in an inpatient setting at a single site and may not be representative of other settings or hospitals. However, parent perceptions regarding influenza vaccine importance for their children are similar in other studies, suggesting that results may be more generalizable. ${ }^{10}$ Only $11 \%$ of parents of children admitted to ACH during each survey period were enrolled in this study, but when parents were available and eligible, less than $1 \%$ declined. Although the sample population represented a small percentage of the admitted population, they were equivalent overall. Minority populations were underrepresented, but other studies have not found significant racial differences in parent perceptions related to influenza vaccination of their children. ${ }^{6}$ Many parents of children admitted to the ICU were not approached because of the critical status of their child, resulting in underrepresentation of this group.

In conclusion, the majority of parents of hospitalized children surveyed felt that influenza vaccination of HCP should be required, suggesting that this is becoming the social norm. This information may help guide hospital policy decisions about required HCP annual influenza vaccination. Additional research is needed to determine whether parents and patients in other healthcare settings have similar expectations of HCP.

\section{ACKNOWLEDGMENTS}

We thank Kathy Thessing and the nurses from the Arkansas Children's Hospital Pediatric Clinical Research Unit for their assistance with the study.
Financial support. This work was supported by the Horace C. Cabe Foundation.

Potential conflicts of interest. All authors report no conflicts of interest relevant to this article. All authors submitted the ICMJE Form for Disclosure of Potential Conflicts of Interest, and the conflicts that the editors consider relevant to this article are disclosed here.

Affiliations: 1. Pediatric Infectious Diseases Section, Department of Pediatrics, University of Arkansas for Medical Sciences, Little Rock, Arkansas; 2. Infection Prevention and Control Department, Arkansas Children's Hospital, Little Rock, Arkansas; 3. Pediatric Biostatistics, Department of Pediatrics, University of Arkansas for Medical Sciences, Little Rock, Arkansas.

Address correspondence to W. Matthew Linam, MD, MS, Department of Pediatrics, University of Arkansas for Medical Sciences, Pediatric Infectious Diseases Section, 1 Children's Way, Slot 512-11, Little Rock, AR 72202 (wlinam@uams.edu).

Presented in part: Pediatric Academic Societies Annual Meeting; Boston, MA; April 28-May 1, 2012 (Abstract 2915.207).

Received March 4, 2014; accepted May 26, 2014; electronically published September 2, 2014.

(C) 2014 by The Society for Healthcare Epidemiology of America. All rights reserved. 0899-823X/2014/3510-0014\$15.00. DOI: $10.1086 / 678061$

\section{REFERENCES}

1. Pearson ML, Bridges $\mathrm{CB}$, Harper SA. Influenza vaccination of health-care personnel: recommendations of the Healthcare Infection Control Practices Advisory Committee (HICPAC) and the Advisory Committee on Immunization Practices (ACIP). MMWR Recomm Rep 2006;55:1-16.

2. Talbot TR, Dellit TH, Hebden J, Sama D, Cuny J. Factors associated with increased healthcare worker influenza vaccination rates: results from a national survey of university hospitals and medical centers. Infect Control Hosp Epidemiol 2010;31:456-462.

3. Influenza vaccination coverage among health-care personnelUnited States, 2012-13 influenza season. MMWR Morb Mortal Wkly Rep 2013;62:781-786.

4. Talbot TR, Babcock H, Caplan AL, et al. Revised SHEA position paper: influenza vaccination of healthcare personnel. Infect Control Hosp Epidemiol 2010;31:987-995.

5. Wellek S. Testing Statistical Hypotheses of Equivalence. Boca Raton, FL: CRC Press, 2003.

6. Daley MF, Crane LA, Chandramouli V, et al. Influenza among healthy young children: changes in parental attitudes and predictors of immunization during the 2003 to 2004 influenza season. Pediatrics 2006;117:e268-e277.

7. Godin G, Vezina-Im LA, Naccache H. Determinants of influenza vaccination among healthcare workers. Infect Control Hosp Epidemiol 2010;31:689-693.

8. Reid AE, Aiken LS. Correcting injunctive norm misperceptions motivates behavior change: a randomized controlled sun protection intervention. Health Psychol 2013;32:551-560.

9. Perkins HW, Berkowitz AD. Perceiving the community norms of alcohol use among students: some research implications for campus alcohol education programming. Int J Addict 1986;21: 961-976.

10. Thors V, Moulsdale P, Finn A. Parental views on childhood influenza vaccination. Pediatr Infect Dis J 2014;33:334-335. 